\title{
Controlador fuzzy para conversor boost de alto rendimento em MCD aplicado a sistema de bombeamento fotovoltaico
}

\author{
Igor R. Sousa \\ Pós-Grad. Eng. de Teleinformática \\ Universidade Federal do Ceará (UFC) \\ Campus do Pici, Centro de Tecnologia \\ igor.sousa@alu.ufc.br
}

\author{
Cláudio M. S. Medeiros \\ Pós-Grad. Energias Renováveis \\ Instituto Federal do Ceará (IFCE) \\ Campus Fortaleza \\ claudiosa@ifce.edu.br
}

\author{
Guilherme A. Barreto \\ Pós-Grad. Eng. de Teleinformática \\ Universidade Federal do Ceará (UFC) \\ Campus do Pici, Centro de Tecnologia \\ gbarreto@ufc.br
}

\begin{abstract}
Neste trabalho, é abordado um projeto de controlador fuzzy de tensão de um conversor CC-CC de alto ganho e alto rendimento de $1 k W$ operando em modo de condução descontínua. Este modo de condução para este conversor específico dificulta a utilização de controladores clássicos. $O$ conversor em questão faz parte de um sistema de bombeamento fotovoltaico autônomo sem baterias composto por quatro painéis fotovoltaicos, um inversor de frequência e um motor de indução acoplado a uma bomba. $O$ conversor boost é responsável pela elevação da tensão de painéis fotovoltaicos para um barramento CC de $311 \mathrm{~V}$, o qual não deve possuir oscilações para um bom funcionamento de um algoritmo de rastreamento do ponto de máxima potência dos painéis. $O$ controlador fuzzy foi implementado em um dispositivo DSP para controle do conversor boost, produzindo uma resposta ao degrau com sobressinal de $5,78 \%$, tempo de acomodação de $1,52 s$ e erro zero em regime permanente.
\end{abstract}

Index Terms-bombeamento fotovoltaico, controle fuzzy, conversor boost.

\section{INTRODUÇÃO}

Sistemas de bombeamento fotovoltaico são geralmente compostos de um conjunto de painéis fotovoltaicos, um barramento CC e um conversor eletrônico para acionamento do conjunto motor-bomba. Para o acionamento de um motor de indução de $220 \mathrm{~V}$, o barramento CC necessita de um tensão de $311 \mathrm{~V}$.

A necessidade de se utilizar muitos painéis fotovoltaicos em série para atingir esta tensão, como em Raju, Kanik e Jyoti [1], faz com que muitos autores utilizem um conversor CC-CC boost, obtendo a tensão desejada com menos painéis. Dentre estes, pode-se destacar o trabalho de Vongmanee [2], que utilizou um conversor CC-CC boost clássico. Porém, o ganho deste conversor é limitado pelas perdas por comutação na chave, diodo e resistência série do capacitor e indutor [3].

Já Ferreira Filho et al. [4] utilizam um conversor CCCC boost com alto ganho de tensão baseado na célula de comutação de três estados (AGT-CCTE) proposto por TorricoBascopé et al. [5]. Uma das principais vantagens deste conversor é a de que as baixas tensões de bloqueio nas chaves permitem a utilização de MOSFETs de baixa resistência, aumentando a eficiência do conversor. Um alto rendimento e um alto ganho estático fazem deste conversor uma opção bastante atrativa para sua utilização com poucos painéis fotovoltaicos em série [5]. Ferreira Filho et al. [4] e Silveira et al. [6] relatam um rendimento deste conversor acima de $93 \%$, para um protótipo de potência de $1 \mathrm{~kW}$.

Com o objetivo de manter constante a tensão de saída do conversor boost independentemente de variações moderadas da tensão de entrada, costuma-se fechar uma malha de controle de tensão com controladores lineares, sejam eles convencionais [7] ou baseados na realimentação de estados [8]. Para o projeto desses controladores é necessária a elaboração de um modelo linear para representar dinamicamente o conversor boost. Uma opção é a formulação de um modelo por função de transferência baseado na teoria dos pequenos sinais [9]. Porém, em alguns casos, principalmente diante de fortes não linearidades, a aplicação dessa técnica ainda não fornece um modelo satisfatoriamente representativo do problema. Assim, a aplicação de controladores não lineares baseado no conhecimento empírico de especialistas, como os controladores fuzzy, torna-se uma alternativa atraente.

No sistema de controle proposto por Ferreira Filho et al. [4], os autores se utilizam de um controlador digital convencional do tipo PI para controlar a tensão de saída do conversor CC boost AGT-CCTE, projetado por Silveira e TorricoBascopé [6]. Neste caso, os autores operam o conversor apenas no modo de condução contínua, o que facilita a elaboração de um modelo linear.

$\mathrm{Na}$ aplicação abordada neste artigo, os autores se deparam com a operação do conversor CC-CC boost AGT-CCTE no modo de condução descontínua (MCD). Esta condição se apresenta durante o processo de inicialização do sistema de bombeamento fotovoltaico e durante os momentos de baixa irradiância ou de falta de água para bombeamento. A operação do conversor CC-CC boost AGT-CCTE em MCD sem que exista um controle específico para este modo pode induzir problemas em outras etapas do sistema de bombeamento fotovoltaico, como oscilações de tensão que impossibilitam um bom MPPT (sigla em inglês para rastreamento do ponto de máxima potência) dos painéis fotovoltaicos.

Este trabalho trata apenas do controle de tensão de saída 
de um conversor CC-CC boost AGT-CCTE em MCD que integra um sistema de bombeamento fotovoltaico. Utiliza-se um sistema de inferência fuzzy para projeto do controlador.

A organização deste trabalho segue a seguinte ordem. A Seção II apresenta a planta real de controle a ser utilizada no trabalho. A Seção III contém informações a respeito do conversor CC-CC boost AGT-CCTE e explicações do porquê controladores convencionais não são uma boa solução para o problema. A Seção IV trata do projeto do controlador fuzzy para o problema. Já a seção $\mathrm{V}$ apresenta os resultados de simulação e resultados reais do sistema de controle. Por fim, a Seção VI descreve as conclusões o trabalho.

\section{Sistema de Controle de Tensão}

A entrada do sistema é constituída de quatro painéis fotovoltaicos CS6K-270 da Canadian Solar ${ }^{\circledR}$. A associação dos quatro painéis é feita da seguinte forma: dois painéis são ligados em série e estão em paralelo com outros dois painéis em série. São utilizados diodos de bloqueio para evitar problemas relacionados com incompatibilidade de tensão entre painéis em paralelo. Os quatro painéis estão posicionados lado a lado e podem ser observados na Figura 1a.

O conversor CC-CC boost AGT-CCTE a ser utilizado é responsável pela elevação da tensão dos painéis para $311 \mathrm{~V}$ do barramento $\mathrm{CC}$ do sistema de bombeamento. O conversor foi construído pelo grupo de pesquisa do Laboratório de Processamento de Energia (LPE) do Instituto Federal de Ciência, Educação e Tecnologia do Ceará (IFCE). Os sinais

Figura 1: Sistema completo de controle do conversor boost.

(a) Painéis fotovoltaicos.

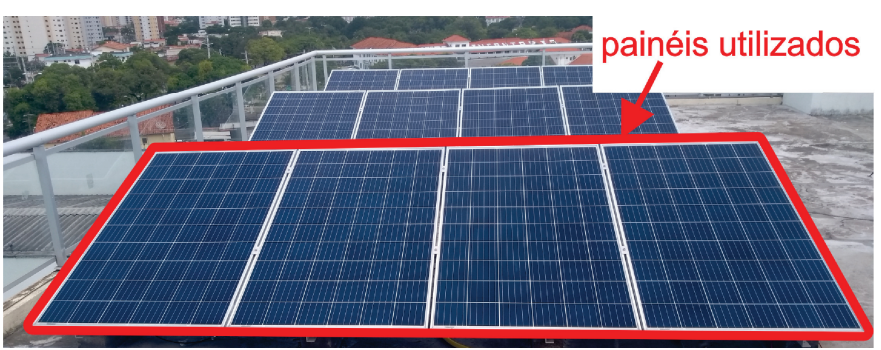

(b) Sistema eletrônico.

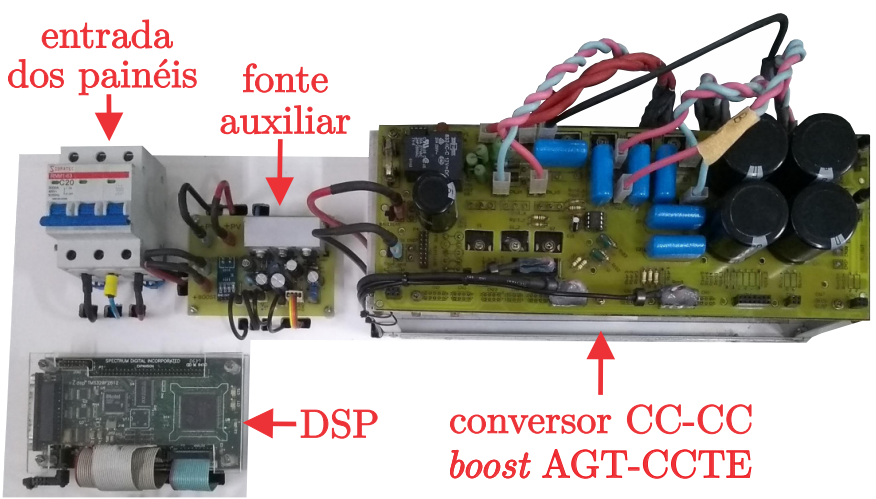

PWMs provenientes do DSP para o comando do conversor são conectados ao driver MC33152p.

O sistema eletrônico montado para este trabalho com base no conversor proposto em [6] está mostrado na Figura 1b. Nesta figura visualiza-se a placa com o DSP, o conversor CCCC boost AGT-CCTE e a entrada dos painéis fotovoltaicos. A fonte auxiliar presente na figura alimenta, a partir dos painéis, o circuito de driver do conversor CC-CC boost e o microcontrolador DSP.

Para realizar o controle de tensão de saída do conversor CCCC boost AGT-CCTE, é utilizado o DSP TMS320F2812 da Texas ${ }^{\circledR}$. As principais características deste DSP que levaram à escolha do mesmo para este trabalho são as seguintes: frequência de clock de $150 \mathrm{MHz}, 16$ portas analógicas multiplexadas em dois conversores A/D de 12 bits, dois gerenciadores de eventos independentes, registrador de soma e produto de 32 bits, e estrutura de hardware para aplicação de modulação por vetores espaciais.

Optou-se pelo uso deste DSP pois, além do controle do conversor boost, o sistema de bombeamento realiza um MPPT (maximum power point tracking) fuzzy dos painéis fotovoltaicos, o acionamento SVPWM (space vector PWM) do inversor de frequência variável, a leitura e filtragem digital de diversos sinais analógicos e a supervisão geral desses processos.

\section{Conversor CC-CC Boost AGT-CCTE}

O conversor CC-CC boost usado neste trabalho utiliza o dimensionamento realizado por Silveira et al. [6], cujo transformador possui dois enrolamentos secundários com relações iguais a 1 . A potência nominal do conversor é de $1 \mathrm{~kW}$. Os autores não recomendam a utilização deste conversor em malha aberta (sem controle de tensão) e sem uma carga conectada à saída, pois a tensão de saída tende a crescer a ponto de danificar os capacitores de saída. Além disso, os sinais PWMs devem possuir frequência de chaveamento de $25 \mathrm{kHz}$ e defasagem entre si de $180^{\circ}$. O diagrama do conversor é apresentado na Figura 2.

Visto que o modo de condução descontínua deste conversor possui resposta com alta não linearidade [10], é realizada uma simulação no PSIM® para obtenção de um modelo de pequenos sinais. As especificações dos componentes do conversor estão descritas na Tabela I. É realizada uma variação de razão cíclica de $7 \%$ para $34 \%$, carga $\mathrm{R}$ de $30 k \Omega$ e tensão de entrada fixa de $65 \mathrm{~V}$. Utilizando informações de máximo sobressinal e tempo de acomodação, pode-se obter um modelo linear de segunda ordem no domínio da frequência. Assim, um modelo de pequenos sinais que relaciona a tensão de saída do conversor CC-CC boost com a razão cíclica aplicada é dimensionado de acordo com a seguinte função de transferência:

$$
G(z)=\frac{V c c(z)}{D(z)}=\frac{0,01233 z+0,0118}{z^{2}-1,858 z+0,8728}+262,23 .
$$


Figura 2: Diagrama do conversor CC-CC boost AGT-CCTE.

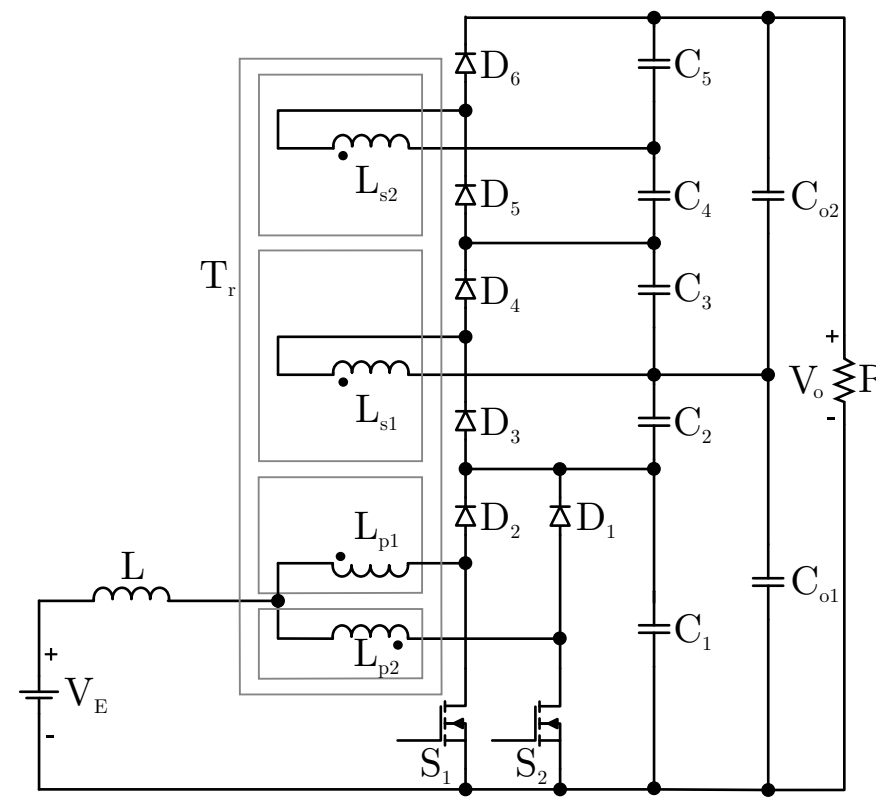

Tabela I: Especificações dos componentes.

\begin{tabular}{c|c|c}
\hline componente & especificação & $\begin{array}{c}\text { resistência } \\
\text { interna }\end{array}$ \\
\hline$D_{1}$ e $D_{2}$ & limiar de $1,7 \mathrm{~V}$ & \multirow{2}{*}{$0,1 \Omega$} \\
\hline$D_{3}, D_{4}, D_{5}$ e $D_{6}$ & limiar de $1,3 \mathrm{~V}$ & \\
\hline$S_{1}$ e $S_{2}$ & queda de $1,3 \mathrm{~V}$ & $0,021 \Omega$ \\
\hline$L$ & $60 \mu \mathrm{H}$ & $0,001 \Omega$ \\
\hline$C_{1} \ldots C_{5}$ & $2,2 \mu \mathrm{F}$ & $0,02 \Omega$ \\
\hline$C_{o 1}$ e $C_{o 2}$ & $470 \mu F$ & $0,230 \Omega$ \\
\hline \multicolumn{2}{|c}{}
\end{tabular}

A comparação entre a resposta do modelo estipulado e do conversor boost simulado à variação de degrau citada é ilustrada na Figura 3. Nota-se que o modelo estipulado não corresponde inteiramente à resposta real do sistema. Isto se deve à natureza não linear da planta. Porém, verifica-se uma boa aproximação para o tempo de acomodação e máximo sobressinal.

É realizada então uma simulação da resposta em malha fechada do modelo usando o controlador PI projetado por Ferreira Filho et al. [4] para o modo de condução contínua. $\mathrm{Na}$ Figura $4 \mathrm{a}$ é ilustrada a resposta para uma pequena variação de referência do controlador (a mesma variação de tensão de saída do conversor para o teste de pequenos sinais da Figura 3). Embora a resposta tenha excelente resultado para uma pequena variação de referência, com tempo de acomodação de $2,86 s$ e sobressinal igual a zero, o mesmo não acontece para grandes variações de referência, como ilustrado na Figura 4b. A resposta possui $18,7 \%$ de sobressinal e tempo de acomodação de $19 \mathrm{~s}$. Estas grandes oscilações de tensão são indesejadas para o sistema em questão.

Analisando o gráfico do lugar das raízes para o modelo da
Figura 3: Respostas do conversor a um degrau de razão cíclica.

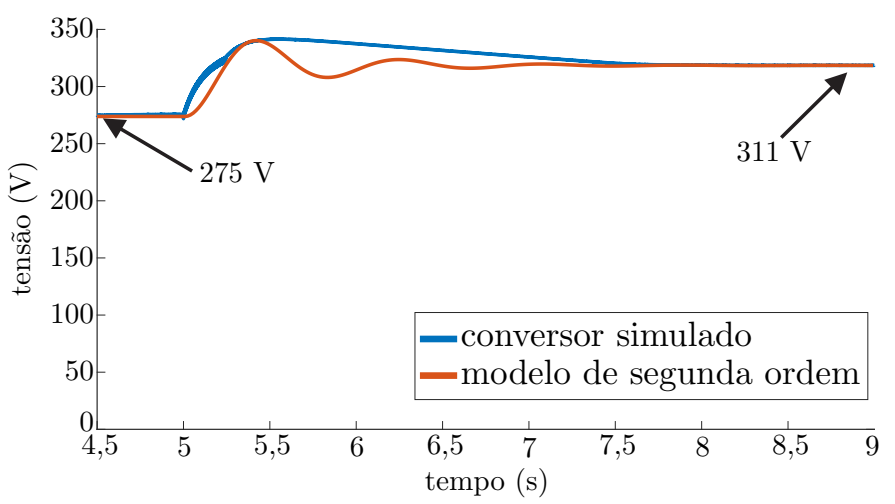

Figura 4: Resposta simulada do conversor ao controlador PI.

(a) Pequena variação de referência do controlador.

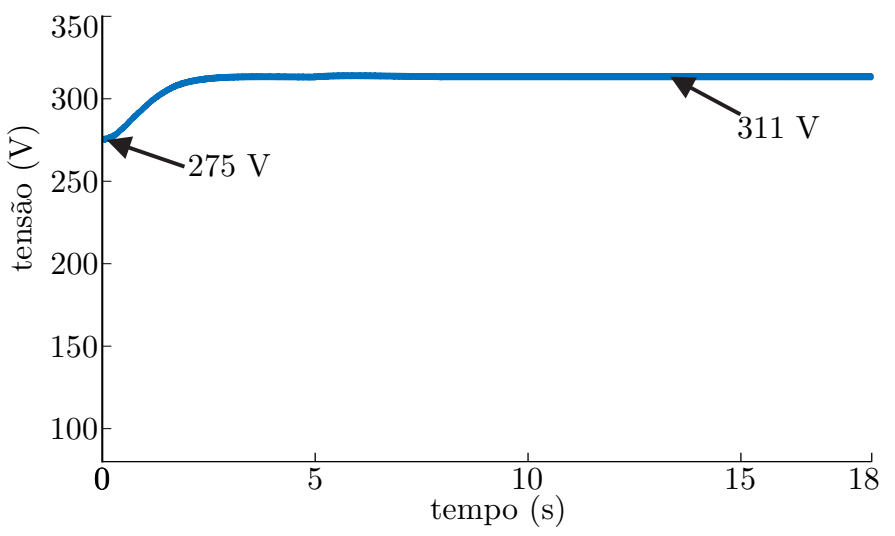

(b) Grande variação de referência do controlador.

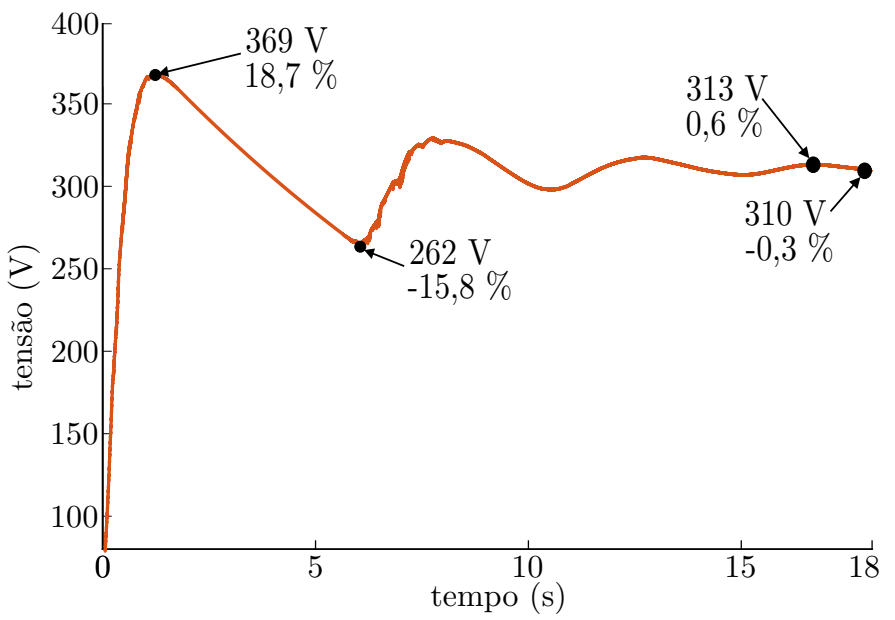

Eq. (1) e o controlador PI de Ferreira Filho et al. [4] mostrado na Figura 5a, observa-se que a maior parte do lugar das raízes se encontra na região de instabilidade, ou seja, fora do círculo unitário. Também pode-se observar que os polos complexos conjugados são dominantes, resultando em sobressinal teórico de $15,6 \%$. Uma vez que a aplicação é a alimentação de um 
Figura 5: Lugar das raízes para controle digital clássico.

(a) Controlador PI com zero em 0,4.

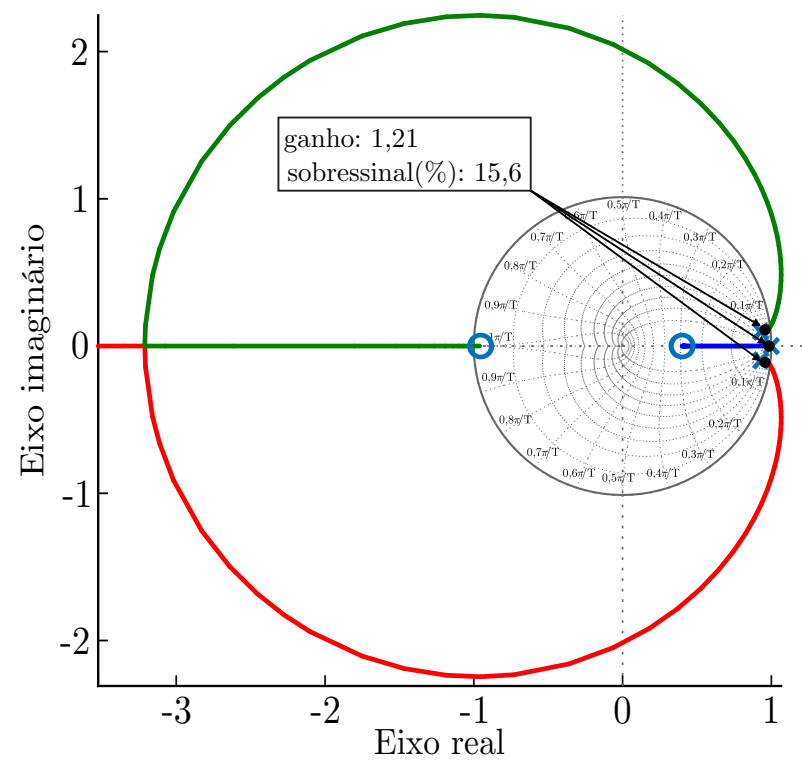

(b) Controlador PID com zeros em 0,8 e 0,9 .

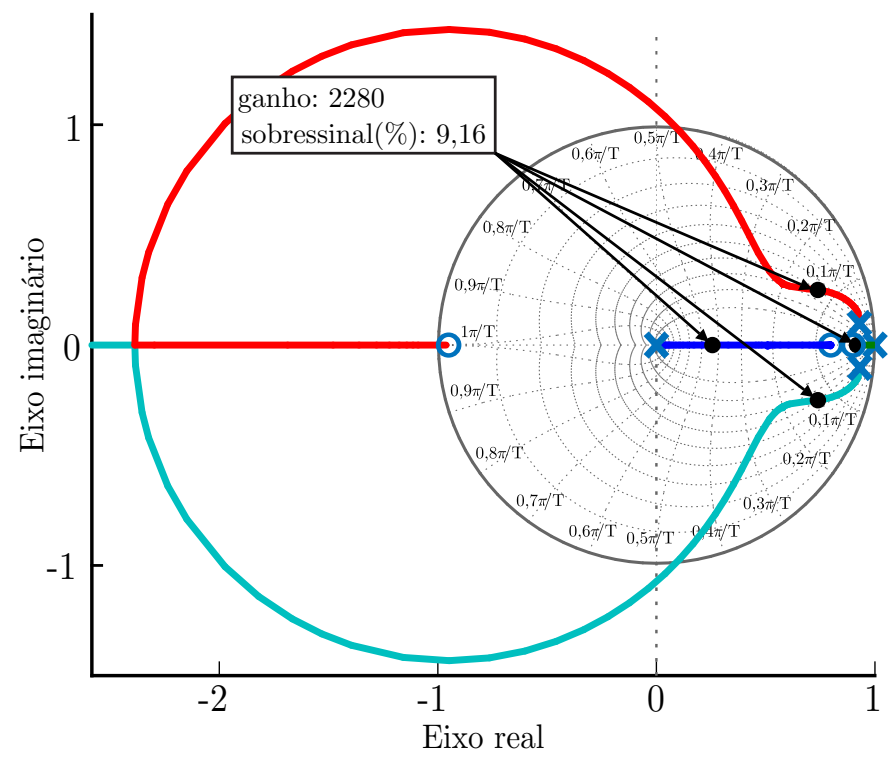

barramento CC para sistema de bombeamento fotovoltaico, deseja-se que o sobressinal seja o mais próximo de zero e sem oscilações. Assim, o controlador PI não atende as necessidades de projeto.

A aplicação de um controlador PID cujo lugar das raízes é ilustrado na Figura 5b, também não obteve bom resultado. Dentre as diversas possibilidades de zeros do controlador, a escolha dos zeros em 0,8 e 0,9 faz com que existam pontos no lugar das raízes em que é possível obter sobressinal de $9,16 \%$ e polo real dominante. Porém, é necessário um ganho de 2280, o que impossibilita a implementação do controlador.

Ao invés de tentar aplicar outros controladores clássicos como o avanço e atraso de fase, optou-se pela utilização de um controlador fuzzy. Uma vez que o sistema se comporta de forma não linear para a condição de MCD, a utilização de um controlador fuzzy é bem atrativa.

\section{PROJETO DO CONTROLAdOR FUZZY}

Entre os tipos clássicos de controlador fuzzy, destacam-se o de Takagi-Sugeno [11] e o controlador de Mamdani [12]. Neste trabalho, é utilizado o controlador do tipo Mamdani. A estrutura deste tipo de sistema de inferência fuzzy é ilustrada na Figura 6, enquanto que o diagrama de blocos do sistema de controle fuzzy deste trabalho é apresentado na Figura 7. Isto posto, de acordo com Perry et al. [13], as entradas utilizadas em um sistema de controle fuzzy em que se deseja que o erro de estado permanente seja igual a zero são geralmente o erro entre o valor desejado e a saída do sistema $(e[k])$ e sua variação $(\Delta e[k])$. Tais entradas do controlador fuzzy deste trabalho são definidas como

$$
\begin{aligned}
e[k] & =\operatorname{Ref}[k]-\operatorname{Sens}[k], \\
\Delta e[k] & =e[k]-e[k-1],
\end{aligned}
$$

em que $\operatorname{Ref}[k]$ é o setpoint de referência discreta e Sens $[k]$ é o sinal de saída discreto do sensor de tensão. O ganho do sensor é definido como $K_{s}=0,007395$ neste trabalho. Já a saída do controlador é a variação de razão cíclica $\Delta d[k]$, e a atualização de $d[k]$ deve ser realizada da seguinte forma:

$$
d[k]=d[k-1]+\Delta d[k] .
$$

Figura 6: Estrutura de um sistema de inferência fuzzy.

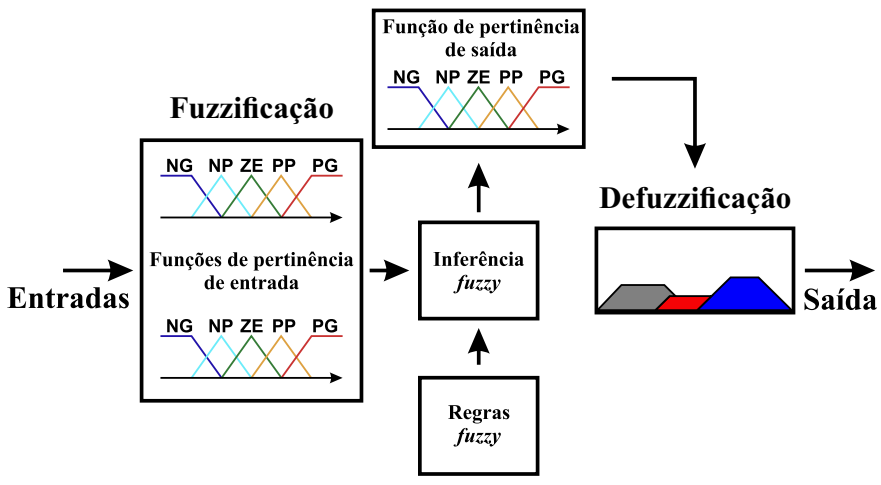

Figura 7: Diagrama de blocos do sistema de controle fuzzy.

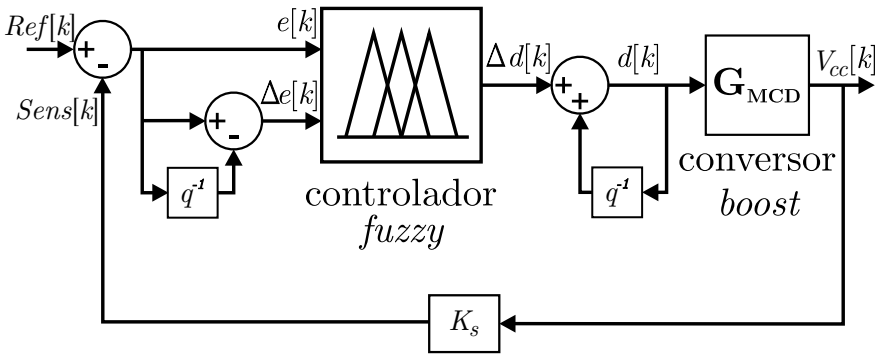


O processo de fuzzificação ilustrado na Figura 6 define uma representação fuzzy para os valores numéricos de entrada. Isto é obtido através das funções de pertinência (FPs) associadas a cada conjunto fuzzy no espaço de entrada. Para cada valor de entrada, são atribuídos graus de pertinência $(\mu)$ de acordo com os conjuntos fuzzy definidos. As FPs podem possuir diversos formatos como triangular, trapezoidal e gaussiana. Porém, sua variação deve ser de 0 a 1, para normalizar as entradas [14].

São escolhidas cinco funções de pertinência (FP) para o universo de discurso de cada entrada e saída fuzzy, possuindo formato triangular pela facilidade de implementação: Negativo Grande (NG), Negativo Pequeno (NP), Zero (ZE), Positivo Pequeno (PP) e Positivo Grande (PG). Após um processo de sintonização, são escolhidas as FPs de entrada e saída do sistema fuzzy, ilustradas na Figura 8.

Já na etapa de inferência, é realizado o mapeamento das entradas fuzzificadas para as regras fuzzy, produzindo uma saída fuzzy para cada regra. Estas regras são baseadas nas funções de pertinência de entrada e ativam funções de pertinências de saída $\mu_{s}$. O grau de pertinência de saída é determinado pelos graus de pertinência das entradas a partir de uma implicação matemática, como a de Zadeh, Mamdami, Larsen ou de Lukasiewicz [15]. As 25 regras fuzzy geradas a partir da inferência utilizam declarações SE-ENTÃO e são apresentadas na Tabela II e ilustradas de forma compacta na Figura 9. Nesta figura, pode-se observar indícios de transições suaves entre as funções de pertinência no universo de discurso de saída.

O grau de pertinência de saída $\mu_{s}\left(\mu_{\Delta d}\right)$ neste trabalho é obtido a partir dos graus de pertinência $\mu_{e}$ e $\mu_{\Delta e}$ das FPs das entradas, com a utilização da implicação de Larsen, ou seja,

$$
\mu_{s_{i}}=\mu_{e_{i}} \cdot \mu_{\Delta e_{i}} .
$$

Como há sobreposição entre as funções de pertinências (vide Figura 8), duas FPs são ativadas para uma mesma entrada, cada uma com seu respectivo grau de pertinência $\mu$. De forma geral, para um sistema de duas entradas, quatro funções de pertinência são ativadas, duas para cada entrada.
Figura 8: FPs dos universos de discurso das variáveis fuzzy.
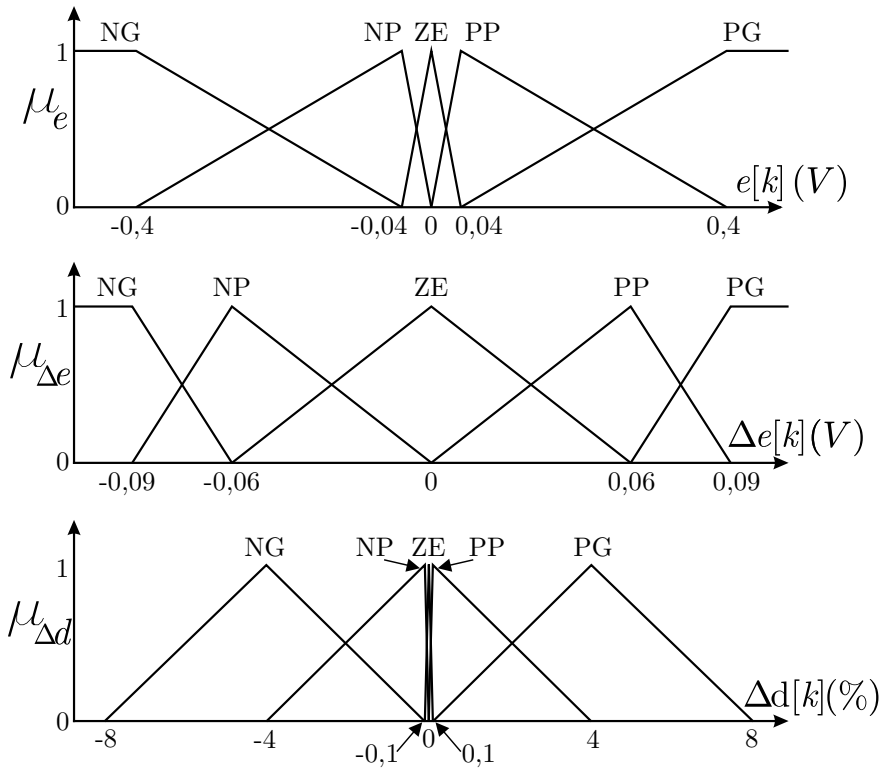

Figura 9: Regras fuzzy simplificada.

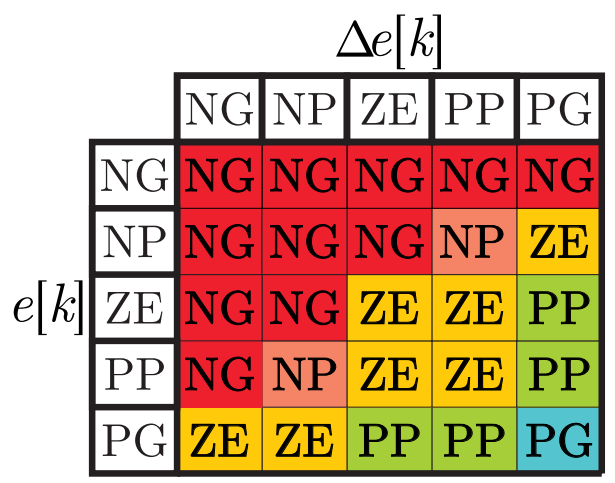

Tabela II: Regras fuzzy do controle de tensão.

\begin{tabular}{|c|c|c|c|c|c|c|c|c|c|c|c|c|c|}
\hline Regra & Se & $e[k]$ & e & $\Delta e[k]$ & Então & Saída & Regra & Se & $e[k]$ & e & $\Delta e[k]$ & Então & Saída \\
\hline $\begin{array}{l}1 \\
2 \\
3 \\
4 \\
\end{array}$ & SE & $\begin{array}{l}\mathrm{NG} \\
\mathrm{NG} \\
\mathrm{NP} \\
\mathrm{NP}\end{array}$ & E & $\begin{array}{l}\text { PP } \\
\text { PG } \\
\text { ZE } \\
\text { PP }\end{array}$ & ENTÃO & $\begin{array}{l}\mathrm{NG} \\
\mathrm{NG} \\
\mathrm{NG} \\
\mathrm{NP} \\
\end{array}$ & $\begin{array}{l}13 \\
14 \\
15 \\
16 \\
\end{array}$ & SE & $\begin{array}{l}\mathrm{NG} \\
\mathrm{NG} \\
\mathrm{NG} \\
\mathrm{NP}\end{array}$ & $\mathrm{E}$ & $\begin{array}{l}\mathrm{NG} \\
\mathrm{NP} \\
\mathrm{ZE} \\
\mathrm{NG} \\
\end{array}$ & ENTÃO & $\begin{array}{l}\mathrm{NG} \\
\mathrm{NG} \\
\mathrm{NG} \\
\mathrm{NG}\end{array}$ \\
\hline $\begin{array}{l}5 \\
6 \\
7 \\
8 \\
\end{array}$ & SE & $\begin{array}{l}\mathrm{NP} \\
\mathrm{ZE} \\
\mathrm{ZE} \\
\mathrm{ZE}\end{array}$ & E & $\begin{array}{l}\mathrm{PG} \\
\mathrm{ZE} \\
\mathrm{PP} \\
\mathrm{PG}\end{array}$ & ENTÃO & $\begin{array}{l}\mathrm{ZE} \\
\mathrm{ZE} \\
\mathrm{ZE} \\
\mathrm{PP}\end{array}$ & $\begin{array}{l}17 \\
18 \\
19 \\
20 \\
\end{array}$ & SE & $\begin{array}{l}\mathrm{NP} \\
\mathrm{ZE} \\
\mathrm{ZE} \\
\mathrm{PP}\end{array}$ & E & $\begin{array}{l}\mathrm{NP} \\
\mathrm{NG} \\
\mathrm{NP} \\
\mathrm{NG}\end{array}$ & ENTÃO & $\begin{array}{l}\mathrm{NG} \\
\mathrm{NG} \\
\mathrm{NG} \\
\mathrm{NG}\end{array}$ \\
\hline $\begin{array}{c}9 \\
10 \\
11 \\
12\end{array}$ & SE & $\begin{array}{l}\text { PP } \\
\text { PP } \\
\text { PP } \\
\text { PG }\end{array}$ & E & $\begin{array}{l}\text { ZE } \\
\text { PP } \\
\text { PG } \\
\text { PG }\end{array}$ & ENTÃO & $\begin{array}{l}\text { ZE } \\
\text { ZE } \\
\text { PP } \\
\text { PG }\end{array}$ & $\begin{array}{l}21 \\
22 \\
23 \\
24 \\
25\end{array}$ & SE & $\begin{array}{l}\text { PP } \\
\text { PG } \\
\text { PG } \\
\text { PG } \\
\text { PG }\end{array}$ & $\mathrm{E}$ & $\begin{array}{l}\text { NP } \\
\text { NG } \\
\text { NP } \\
\text { ZE } \\
\text { PP }\end{array}$ & ENTÃO & $\begin{array}{l}\text { NP } \\
\text { ZE } \\
\text { ZE } \\
\text { PP } \\
\text { PP }\end{array}$ \\
\hline
\end{tabular}


Por fim, a última etapa de um controlador fuzzy é a defuzzificação. A tarefa do processo de defuzzificação é converter a saída das regras fuzzy em um valor escalar a ser aplicado ao processo de controle [14]. Existem vários métodos para encontrar um valor escalar aproximado para representar a ação a ser tomada como o método do max-min, método das médias, método do somatório da raiz quadrada e o método do centroide. $\mathrm{O}$ método de defuzzificação utilizado neste trabalho é o do centroide, no qual as saídas são ponderadas pelas áreas das FPs de saída de acordo com a seguinte expressão:

$$
\Delta d[k]=\frac{\sum_{i=1}^{4} \Delta d(i) \operatorname{Area}(i)}{\sum_{i=1}^{4} \operatorname{Area}(i)},
$$

em que $\Delta d(i)$ é a saída referente ao $i$-ésimo grau de pertinência de saída $\mu_{s i}$ e $\operatorname{Area}(i)$ é a área formada entre $\mu_{s i}$, a $i$-ésima FP de saída ativada e o eixo das abcissas.

\section{RESUltados}

Os resultados de simulação da aplicação do controlador fuzzy ao sistema são reportados na Figura 10 e na Tabela III. $\mathrm{O}$ período de atualização dos controladores é de $16 \mathrm{~ms}$. Podese observar que existe um sobressinal de $0,5 \%$ na resposta do sistema ao controlador fuzzy, em comparação aos 18,7\% com o controlador PI clássico. O sobressinal do controlador fuzzy é considerado aceitável para as especificações de projeto. Nota-se também que não há oscilações como a resposta do PI.

Além do sobressinal ser bem menor, a resposta do sistema ao controlador fuzzy possui tempo de acomodação também bastante inferior. De fato, o tempo de acomodação com o controlador fuzzy é de $1,1 \mathrm{~s}$, enquanto que para o controlador PI utilizado é de $19 \mathrm{~s}$. Como já mencionado, as grandes oscilações de tensão presentes na resposta com o controlador PI são indesejadas para o sistema de bombeamento fotovoltaico em questão. O controlador fuzzy também apresenta desempenho superior ao controlador PI neste quesito para esta aplicação.

As saídas dos controladores (razão cíclica) simulados são ilustradas na Figura 10b. Pode-se observar que durante um período de $3,75 \mathrm{~s}$, a saída do controlador PI é saturada em $0 \%$, exibindo um comportamento não desejável. Nota-se também grandes oscilações. Já na saída do controlador fuzzy, percebese que estas oscilações não existem e que não há saturação.

A trajetória das ativações são ilustradas na Figura 11. Notase que existe uma convergência na trajetória das ativações, o que evidencia uma estabilidade do sistema. Porém, nem todas as regras são utilizadas. De fato, somente 12 das 25 regras são ativas. Estas regras ativadas estão exibidas no lado esquerdo da Tabela II, numeradas de 1 a 12 .

Os resultados das implementações dos controladores fuzzy e PI clássico no sistema real da Figura 1 são apresentados na Figura 12 e Tabela IV. Percebe-se que no sistema real com controlador fuzzy, existe um sobressinal de $5,78 \%$, o que faz a resposta ter um pico de $329 \mathrm{~V}$. Porém, o período transitório possui apenas $3,8 s$ de duração, ou seja, não existem grandes oscilações. Já no sistema real com controlador PI, o sobressinal
Figura 10: Respostas simuladas do sistema aos controladores.

(a) Tensão controlada de saída.

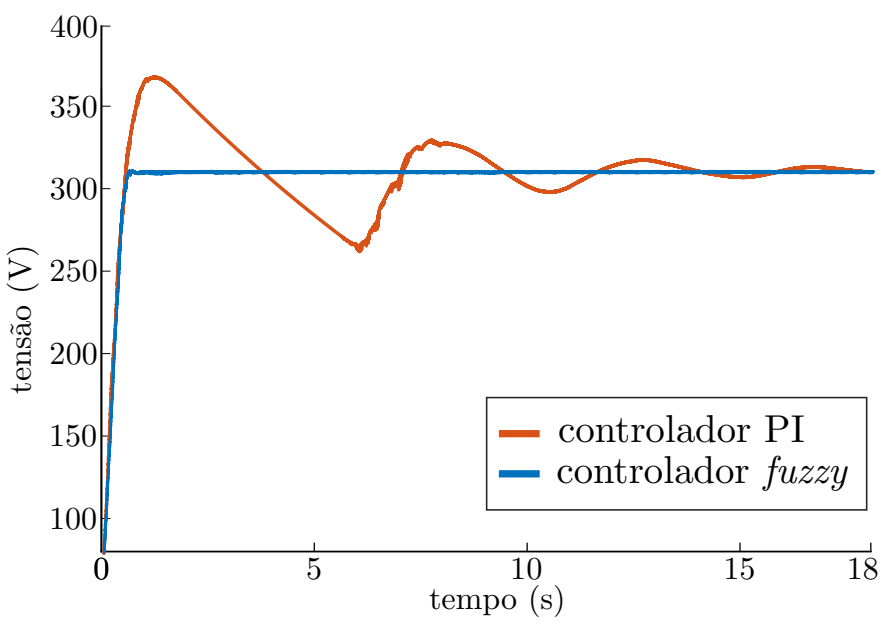

(b) Saída dos controladores.

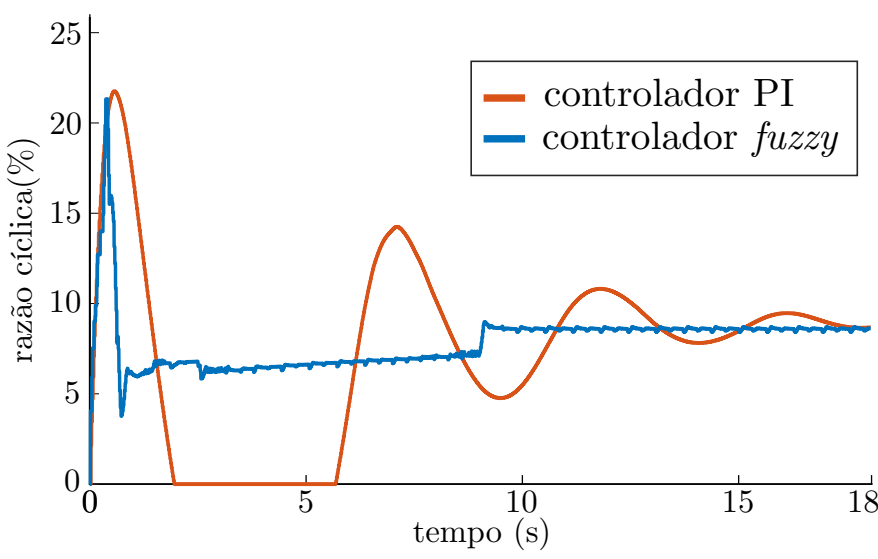

Tabela III: Métricas das respostas do sistema simulado.

\begin{tabular}{c|c|c|c}
\hline controlador & sobressinal & subressinal & $\begin{array}{c}\text { tempo de } \\
\text { acomodação }\end{array}$ \\
\hline PI clássico & $\begin{array}{c}18,7 \% \\
(369 \mathrm{~V})\end{array}$ & $\begin{array}{c}-15,8 \% \\
(262 \mathrm{~V})\end{array}$ & $1,1 \mathrm{~s}$ \\
fuzzy & $0,5 \%$ & $-0,2 \%$ & $19 \mathrm{~s}$ \\
& $(311,7 \mathrm{~V})$ & $(310,4 \mathrm{~V})$ & \\
\hline
\end{tabular}

é de $11,2 \%$, com pico de $346 \mathrm{~V}$. O período transitório possui 16 segundos de grandes oscilações.

Após testes reais de bombeamento, o controlador PI clássico para controle do conversor em MCD mostrou-se inadequado, impossibilitado a boa atuação do algoritmo de MPPT dos painéis fotovoltaicos. Para as necessidades do sistema de bombeamento fotovoltaico, o controlador fuzzy de tensão de saída do conversor CC-CC boost AGT-CCTE projetado é considerado satisfatório, tendo em mente os requisitos da aplicação real. 
Figura 11: Trajetória das ativações de regras do controlador.

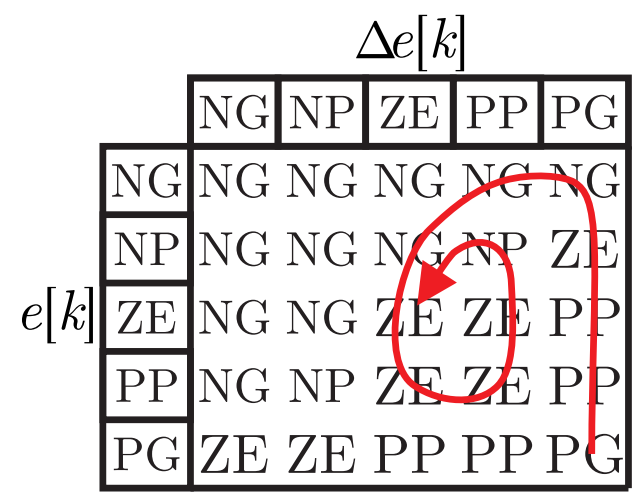

Figura 12: Respostas reais do sistema aos controladores.

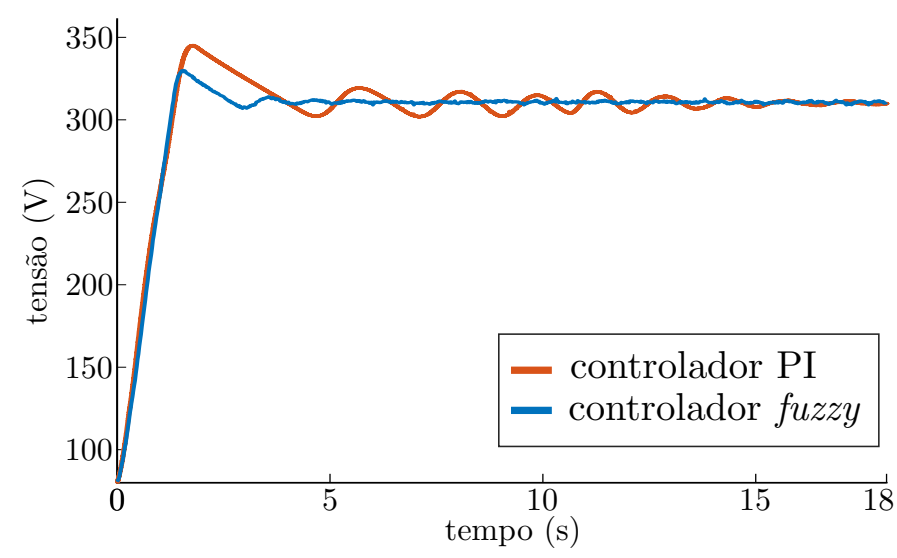

Tabela IV: Métricas das respostas do sistema real.

\begin{tabular}{c|c|c|c}
\hline controlador & sobressinal & subressinal & $\begin{array}{c}\text { tempo de } \\
\text { acomodação }\end{array}$ \\
\hline \multirow{2}{*}{ PI clássico } & $\begin{array}{c}11,2 \% \\
(346 V)\end{array}$ & $\begin{array}{c}-2,57 \% \\
(303 \mathrm{~V})\end{array}$ & $16 \mathrm{~s}$ \\
fuzzy & $\begin{array}{c}5,78 \% \\
(329 \mathrm{~V})\end{array}$ & $\begin{array}{c}-1,6 \% \\
(306 \mathrm{~V})\end{array}$ & $3,8 \mathrm{~s}$ \\
\hline
\end{tabular}

\section{Conclusões}

A utilização de um controlador convencional para conversor em MCD acarreta em grandes oscilações na tensão do barramento CC (vide Figura 12) do sistema de bombeamento. Estas oscilações são refletidas para os painéis, e podem influenciar negativamente o funcionamento de um algoritmo MPPT. A utilização do controlador fuzzy para a região de MCD reduziu substancialmente a amplitude e duração das oscilações de tensão do barramento CC. Sem esta mudança de controlador, as grandes oscilações de potência dos painéis poderiam inviabilizar o funcionamento correto de um MPPT no sistema de bombeamento.

A saída de controle fuzzy não possui oscilações como a do controlador PI e não há saturação. A trajetória das ativações das regras fuzzy mostra que o sistema é estável. A resposta real do sistema com controlador fuzzy possui sobressinal de $5,78 \%$ (329 $\mathrm{V}$ ) e tempo de acomodação de $3,8 \mathrm{~s}$. Para um sistema de bombeamento com MPPT, o controlador de tensão fuzzy do conversor CC-CC boost AGT-CCTE mostrou-se satisfatório para o modo de condução descontínua.

\section{AgRAdECIMENTOS}

O presente trabalho foi realizado com apoio da CAPES (Código de Financiamento 001) e do CNPq (No. 309379/20199).

\section{REFERÊNCIAS}

[1] A. Raju, S. R. Kanik, and R. Jyoti, "Maximum efficiency operation of a single stage inverter fed induction motor PV water pumping system," in 2008 First International Conference on Emerging Trends in Engineering and Technology, 2008, pp. 905-910.

[2] V. Vongmanee, "The photovoltaic pumping system using a variable speed single phase induction motor drive controlled by field oriented principle," in The 2004 IEEE Asia-Pacific Conference on Circuits and Systems, 2004. Proceedings., vol. 2, 2004, pp. 1185-1188.

[3] R.-J. Wai, C.-Y. Lin, R.-Y. Duan, and Y.-R. Chang, "High-efficiency DC-DC converter with high voltage gain and reduced switch stress," IEEE Transactions on Industrial Electronics, vol. 54, no. 1, pp. 354364, 2007.

[4] J. R. M. Ferreira Filho, F. R. F. Mendes, I. R. Sousa, J. R. B. Sousa, and C. M. S. Medeiros, "Photovoltaic panel based pumping system: A solution without batteries," IEEE Latin America Transactions, vol. 16, no. 2, pp. 514-520, 2018.

[5] G. V. Torrico-Bascopé, S. A. Vasconcelos, R. P. Torrico-bascope, F. L. Antunes, D. S. De Oliveira, and C. G. Branco, "A high step-up DC-DC converter based on three-state switching cell," in 2006 IEEE International Symposium on Industrial Electronics, vol. 2, 2006, pp. $998-1003$.

[6] G. C. Silveira, F. L. Tofoli, L. D. S. Bezerra, and R. P. Torrico-Bascopé, "A nonisolated DC-DC boost converter with high voltage gain and balanced output voltage," IEEE Transactions on Industrial Electronics, vol. 61, no. 12, pp. 6739-6746, 2014.

[7] A. Mamizadeh, N. Genc, and R. Rajabioun, "Optimal tuning of PI controller for boost DC-DC converters based on cuckoo optimization algorithm," in 2018 7th international conference on renewable energy research and applications (ICRERA). IEEE, 2018, pp. 677-680.

[8] J. C. Mayo-Maldonado, J. C. Rosas-Caro, R. Salas-Cabrera, A. González-Rodriguez, O. F. Ruíz-Martínez, R. Castillo-Gutiérrez, J. R. Castillo-Ibarra, and H. Cisneros-Villegas, "State space modeling and control of the DC-DC multilevel boost converter," in 2010 20th International Conference on Electronics Communications and Computers (CONIELECOMP), 2010, pp. 232-236.

[9] M. Leng, G. Zhou, Q. Tian, G. Xu, and F. Blaabjerg, "Small signal modeling and design analysis for boost converter with valley $V^{2}$ control," IEEE Transactions on Power Electronics, vol. 35, no. 12, pp. $13475-13487,2020$.

[10] I. R. Sousa, "Sistema de bombeamento fotovoltaico com conversores eletrônicos integrados." Master's thesis, Instituto Federal de Educação, Ciência e Tecnologia do Ceará, 2020.

[11] T. Takagi and M. Sugeno, "Fuzzy identification of systems and its applications to modeling and control," IEEE transactions on systems, man, and cybernetics, no. 1, pp. 116-132, 1985

[12] E. H. Mamdani and S. Assilian, "An experiment in linguistic synthesis with a fuzzy logic controller," International journal of man-machine studies, vol. 7, no. 1, pp. 1-13, 1975.

[13] A. G. Perry, G. Feng, Y.-F. Liu, and P. C. Sen, "A design method for PI-like fuzzy logic controllers for DC-DC converter," IEEE transactions on industrial electronics, vol. 54, no. 5, pp. 2688-2696, 2007.

[14] A. P. Engelbrecht, Computational intelligence: an introduction. John Wiley \& Sons, 2007.

[15] H. B. Verbruggen, H.-J. Zimmermann, and R. Babuška, Fuzzy algorithms for control. Springer Science \& Business Media, 2013, vol. 14. 